\title{
Progress in Titanium Machining
}

\author{
HAAS Franz $^{1, a^{*}}$, ZOPF Philipp ${ }^{2, b}$, and EDLER Jörg ${ }^{3, \mathrm{c}}$ \\ ${ }^{1,2,3} \mathrm{Graz}$ University of Technology, Institute of Production Engineering \\ Kopernikusgasse 24/I, $8010 \mathrm{Graz}$, Austria

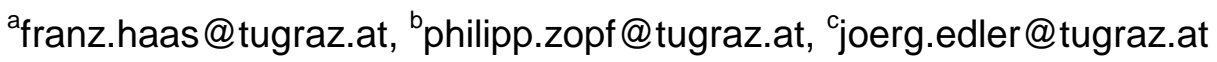

Keywords: Titanium alloys, Milling tools, Ultrasonic machining, Aircraft industry

\begin{abstract}
Milling and drilling of titanium alloys represent a key technology for the aircraft manufacturers. The balancing act between production costs and tool costs leads to the need of tests and an optimized setup of the whole process. A Styrian consortium with experts in materials, tools and machining has been formed to extend the tool life in machining of titanium alloys. A series of tests is set up to evaluate the roughing and finishing operations. For finishing operations ultrasonic assisted milling is introduced and compared with conventional milling. Force measurement and optical wear detection are used for tool characterization.
\end{abstract}

\section{Initial Situation}

Titanium alloys are created by the variation of the structure phases and therefore they can be distinguished into $\alpha$-titanium, $\beta$-titanium and $(\alpha+\beta)$-titanium. Typical elements for the $\beta$-phase are iron, chrome, vanadium, molybdenum and niobium to apply the alloys within a wide temperature range. On the other hand aluminum, tin, oxygen and zircon enlarge the $\alpha$-phase. $(\alpha+\beta)$-titanium alloys allow hardening by a special heat treatment and heat ageing between $450{ }^{\circ} \mathrm{C}$ and $650{ }^{\circ} \mathrm{C}$. A typical $(\alpha+\beta)$-alloy is Ti6Al4V with the short name Ti64.

Chipping of titanium alloys especially with geometrical defined cutting edge is commonly seen as a critical process. The thermomechanical properties of this group of materials, especially the high specific strength by a very low breaking elongation ( $\mathrm{A}=0.5 \%$ to $15 \%)$ combined with a very low thermal conductivity are the challenges for milling or chipping in general. Only $20 \%$ of the resulting heat can be transported by the chip itself, therefore the thermal stability of the cutting material has high importance [1]. The internal direct cooling via small holes at the cutting edges of a milling tool represents a very effective technique that enables an increase of $200 \%$ material removal rate by only $23 \%$ loss of tool life compared with a standard tool [2]. During milling the chips are created segmentally at temperatures of about $900{ }^{\circ} \mathrm{C}$ causing the high mechanical and thermic dynamic load of the tool.

The optimal design of carbide metal inserts for titanium machining has to consider three criteria, the carbide itself, the coating and the geometry. An innovative tool for titanium turning is presented by Böhlerit [3]. The carbide substrate is extremely temperature stable with low tendency to ductile deformation. In addition the substrate is coated with a $3.0 \mu \mathrm{m}$ thick TiBN layer using Plasma CVDprocess. Coating requirements for titanium are both stability against high temperatures and oxidation and low tendency of chip adhension. The third aspect is the geometry with the need of sharp edges and special chip former surface structure. 


\section{Ultrasonic-assisted machining process}

Ultrasonic-assisted milling (UAM) has been developed during the recent years as a method that combines ultrasonic vibrations with the conventional milling process. In [4] the tool life in ultrasonic-assisted milling and conventional milling has been compared. One-dimensional ultrasonic vibrations have been applied on workpiece in the feed direction of tool. Due to tool rotation the direction of workpiece vibrations with respect to the edge of cutting tool varies in time. This causes improper and uncontrolled contacts between tool and workpiece. Impact force between two bodies is proportional to second power of impact velocity of two bodies. The relative velocity of workpiece in direction normal to tool flank surface can be used to estimate the contacts between tool and workpiece. Negative values indicate contact between the newly machined surface and tool flank surface. Calculations show the higher the rotational speed the lower the mentioned negative values. This causes a decreasing number of workpiece impacts. Advantages compared to conventional milling (CM) are listed below: [4]

- Less contacts at higher spindle speed values.

- Lower temperature in the cutting zone.

- Less plastic deformation.

- Change of friction coefficient from semistatic to dynamic mode.

Heavy duty machining mostly benefits from the use of cutting fluids. Corresponding costs (fluid, handling, storage, removal) represent an increasing amount of the total machining costs. Dry machining on the other hand causes higher cutting forces, low surface quality and poor dimensional accuracy. Thermally enhanced ultrasonically assisted machining represents a technology that promises benefits in machining of titanium alloys. It utilizes the effect of shear strength decrease and reduced strain hardening rate at higher temperatures [5]. In the case of a simple turning process hot ultrasonic assisted turning (HUAT) shows some advantages for titanium alloys:

- Significant reduction of cutting forces (up to $80 \%$ ).

- Improvement of surface quality.

- Reduction of stress affected zone within the workpiece.

\section{Machine Description}

The experiments were carried out with a 5-axis-milling center from DMG SAUER (US30 linear). The machine concept is a gantry system with internal cooling of the machine bed for high precision milling and grinding (Fig. 1). The focus lies on surface qualities $\mathrm{Ra}<0,1 \mu \mathrm{m}$. The high dynamic linear motors with active cooling in $\mathrm{X} / \mathrm{Y} / \mathrm{Z}$ enable accelerations of more than $1,2 \mathrm{~g}$ and fast motions of $50 \mathrm{~m} / \mathrm{min}$. The $19 \mathrm{~kW}$ spindle with HSK-E40 interface supplies $40.000 \mathrm{~min}^{-1}$ maximum speed.

For high precision parts made of e.g. ceramics, glass, sapphire the benefits are listed below:

- Process force reduction causes low surface damaging.

- Realization of thin fillets smaller than $0,5 \mathrm{~mm}$.

- Excellent surface quality $(\mathrm{Ra}<0,1 \mu \mathrm{m})$

- Feasibility of deep hole drilling up to 25 times hole diameter

In the field of CFK the technology utilizes additional advantages:

- $40 \%$ lower process forces and the avoidance of delamination.

- Two times higher feed rates.

- Optimal removal of the particles.

- Sharp edges. 
The machine offers three special features to improve ultrasonic machining. Adaptive Control (ADC) means the automatic adjustment of the feed rate measuring the spindle torque within the control circuit. That prevents tool or workpiece damaging and ensures process capability. EASYSONIC-Control is the frequency scanning to detect the resonance frequency as optimal working parameter. Automatic amplitude regulation allows programming of a special amplitude cycle within the machining task. For instance the amplitude has a low values during start and exit phase of a drilling process, a high value is determined during the drilling phase.
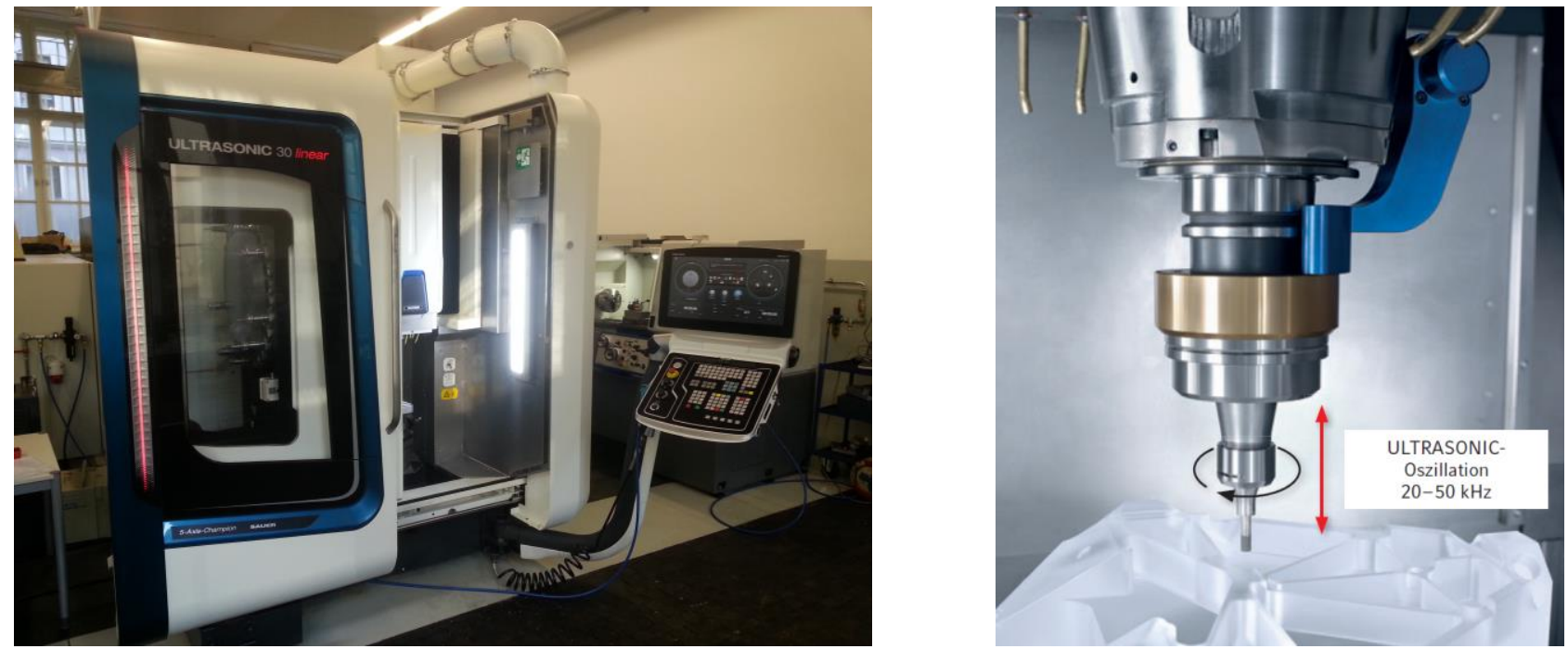

Figure 1: Ultrasonic milling machine (DMG SAUER) at the Institute of Production Engineering.

A detailed description of possible ultrasonic machine arrangements is given in [6]. Examples are ultrasonic erosion machines that are used to cut brittle materials ore special designed machines for deep holes drilling. Other arrangements concern an ultrasonic machine used for wire drawing or an ultrasonic welding machine. Basically two directions concerning the influence of ultrasonics to processes can be distinguished, first the effect of plastic deformation and second the impact of dry friction.

\section{Laser Vibrometer Measurement}

To investigate the machine characteristics in detail a laser vibrometer was used to analyze actual tool holder movements. Therefore the laser source was adjusted outside the working space and a mirror oriented $45^{\circ}$ to the XY-plane deflects the laser beam towards a reflector foil fixed to the plane surface of the tool holder. The high frequent movement is analyzed by the reflecting beam. Interesting values are the velocity orthogonal to the reflecting surface and in addition the FFT calculates the main frequencies. Figure 2 shows both the sketch of the test setup and the real experiment. The preset values for the measurement (sample rate $250.000 \mathrm{~s}^{-1}$ ) illustrated at Figure 3 are the frequency of $51100 \mathrm{~Hz}$ and the maximum possible amplitude (100\%). The measurement result of $51086 \mathrm{~Hz}$ matches with the nominal frequency, the amplitude of approximately $3,2 \mu \mathrm{m}$ is also a realistic value. 

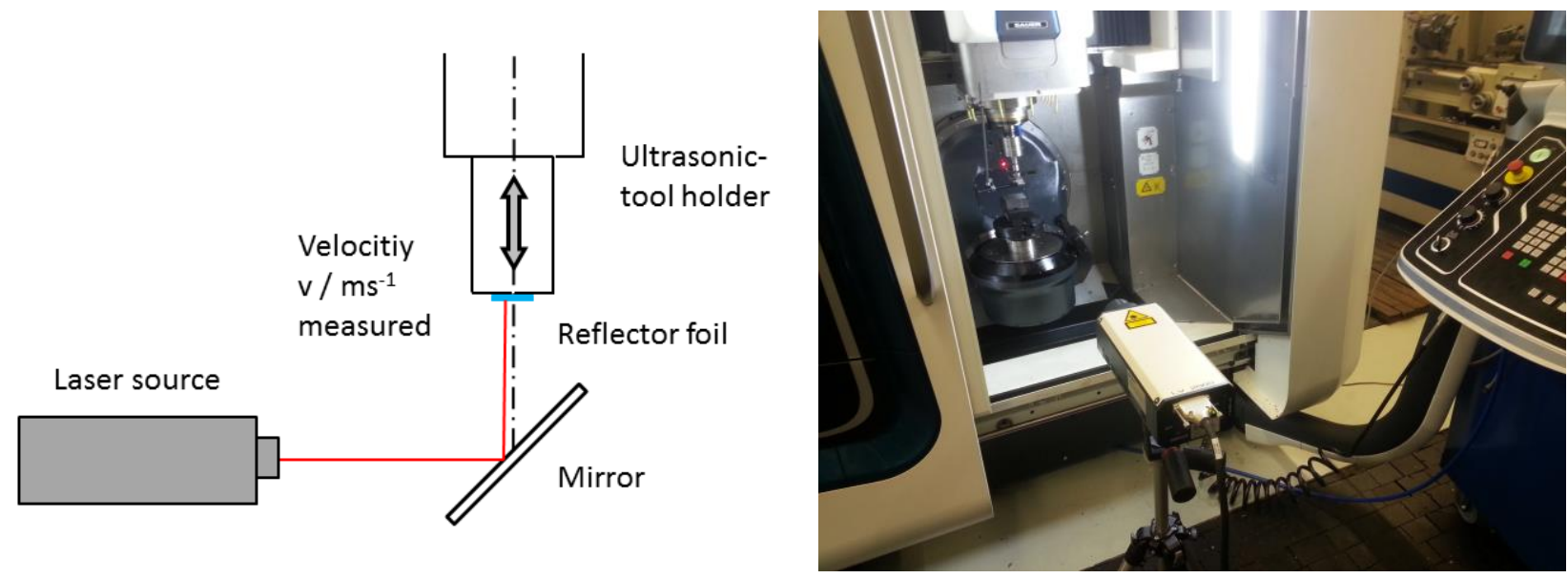

Figure 2: Principle of laser-vibrometer-measurement (left), test setup (right).

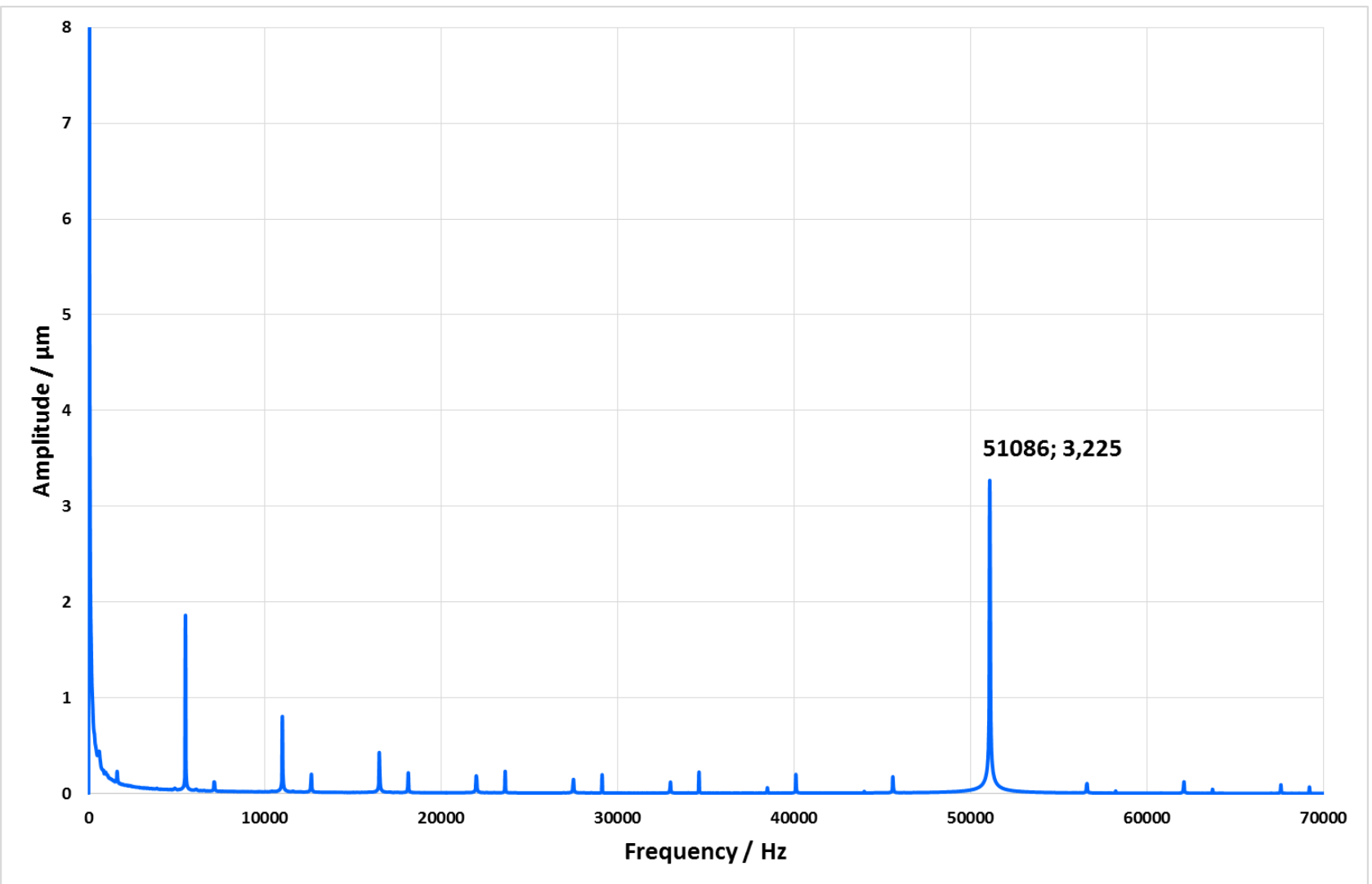

Figure 3: Fast Fourier analysis of the ultrasonic tool holder in vertical direction.

\section{Titanium Milling - Tests}

As a first step the consortium decided to perform benchmark tests with the intention to evaluate the machinability of the test material. With regard to the ultrasonic technology the tests should be done both with and without vibration assistance. The program is divides into rough and finish machining. Milling of flutes and edges represents the rough tests with insert milling tools, the finishing operations are carried out with carbide end mill cutters. All experiments are performed with tools from different manufacturers and the generated experience represents the base for optimized parameters for ultrasonic milling of titanium alloys and the development of new tool geometries.

One tool (rough and finish operations) is defined as a reference for the subsequent comparison concerning tool life and performance. The reference tool will be tested at first and the flank wear will be determined after a defined cutting time at special milling parameters. In the next stage all other tools will be examined by the same cutting parameters and afterwards the resulting wear will 
be measured. Thus a significant rating and evaluation is possible with all chances to learn from the best. Especially the up to now not documented behavior of the tools under ultrasonic condition is from high interest.

To increase the value of the experiments in addition the milling force components are measured. To do this a measurement platform from Kistler is used as specimen fixation to pick up the force components in $\mathrm{X}, \mathrm{Y}$ and Z-direction. The spindle torque cannot be directly detected with the described setup. Especially concerning the vibration assisted technology differences of the forces will be expected.

As it is necessary to modify the tool holder for making ultrasonic tests with rough machining, the program starts with smoothing operations. The cylindrical titanium workpiece is connected to a Kistler platform that is mounted on the machine table. One circumference will be machined within one cycle. The depth of cut is only $0.1 \mathrm{~mm}$ therefore the friction part is extremely high in relation to the total cutting force. The machined surface rises up with decreasing diameter to avoid the influence of a vertical force component. Because of the fact that tool life is longer than 600 minutes relatively long test durations have to be accepted.

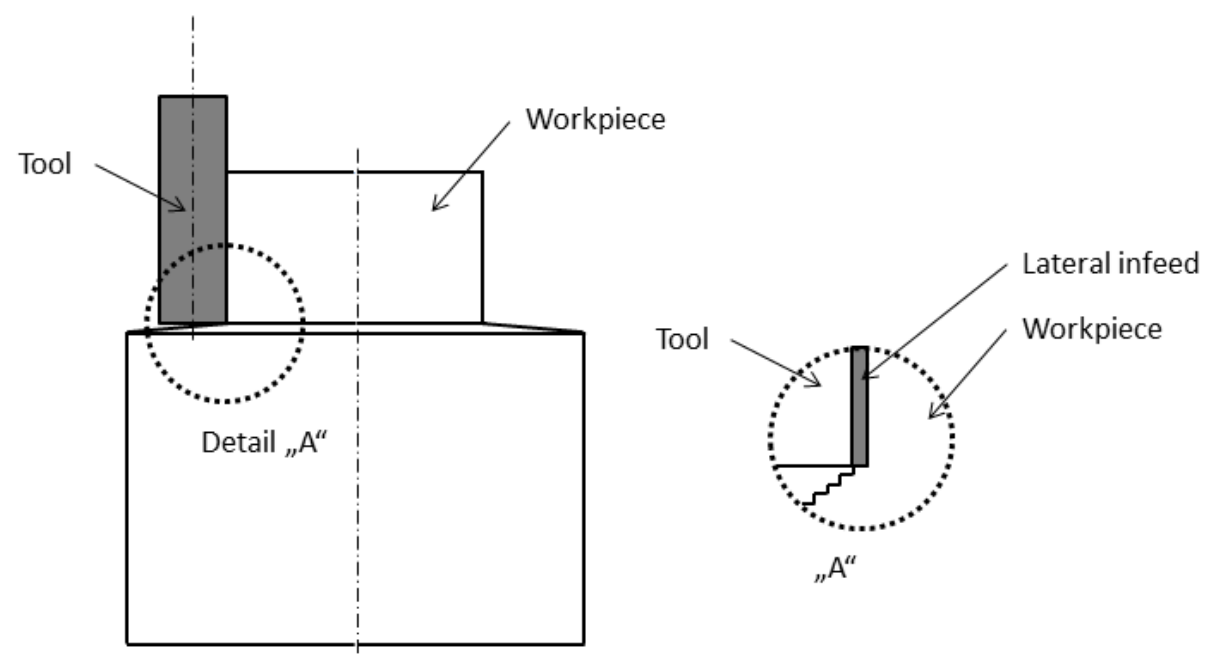

Figure 4: Contact situation of titanium finishing test operation.

Figure 5 gives an overview to the programmed path. Beginning outside the milling tool moves to the contact zone tangentially and fulfils then one entire circle. After that the tool leaves in a tangential way and comes to the end point. During the following cycle the cut of depth $(0.1 \mathrm{~mm})$ is considered. The active force as the vector sum of the components $F_{x}$ and $F_{y}$ is shown in Figure 6. Each cycle can be separated and as main conclusion the mean active force without ultrasonic is about $6 \%$ higher than the mean value picked up with $100 \%$ intensity ultrasonic assistance. The main reason for this is the lower friction applying ultrasonic assistance. Further investigations show the influence of frequency and amplitude.

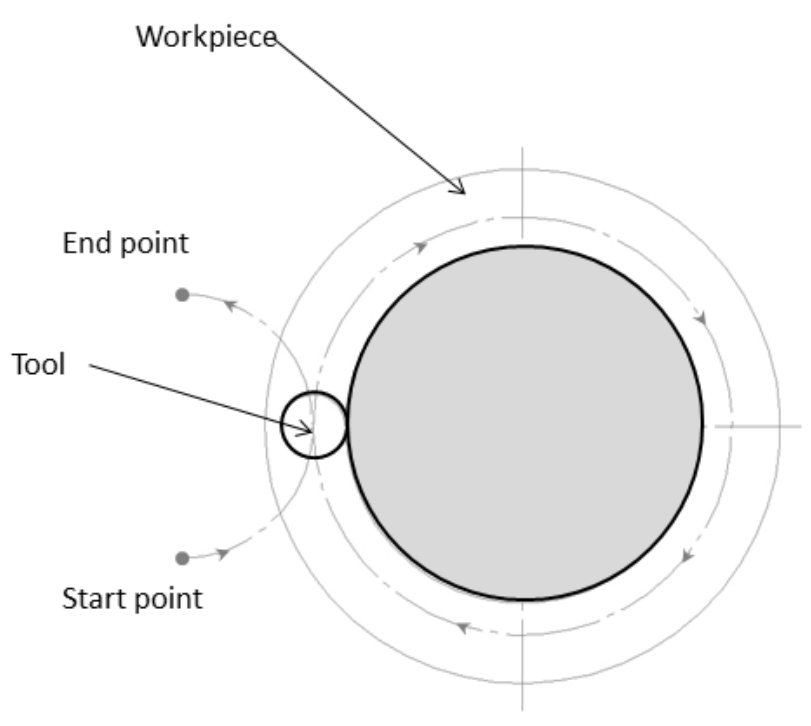

Figure 5: Test sequence for finishing operation. 


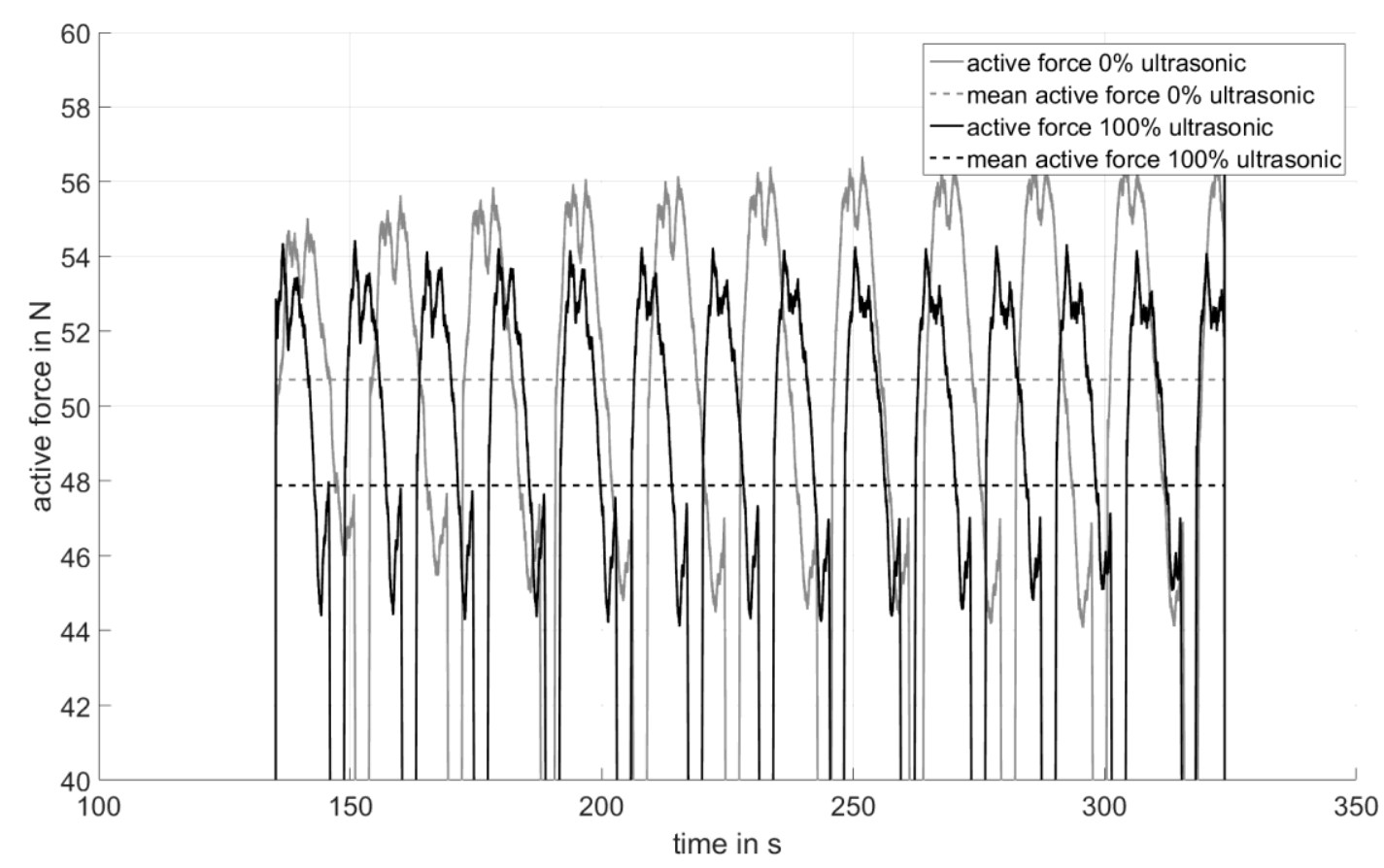

Figure 6: Active force vs. cutting time - conventional milling and ultrasonic assisted milling.

\section{Summary}

Summarizing a single part of an ongoing project to progress titanium machining is presented. The multidisciplinary consortium consisted of both specialists in the field of material, carbide, coating, measurement and applicants are working together to develop new tools for titanium milling. The ultrasonic technology as a rather new and not well established method has potential to support this process.

\section{References}

[1] U. Heisel, F. Klocke, E. Uhlmann, G. Spur, Handbuch Spanen, Hanser, Munich, (2014) 12741279.

[2] B. Denkena et al., Das optimale Hartmetall zum Schruppfräsen von Titan, in Schweizer Präzisions-Fertigungstechnik, (2015) 22-24.

[3] Information on http://www.boehlerit.com.

[4] J. Janghorbanian et al., Effect of cutting speed on tool life in ultrasonic-assisted milling process, in Journal of Engineering Manufacture, (2013) 1157-1164.

[5] R. Muhammad et al., Thermally enhanced ultrasonically assisted machining of Ti alloy, submitted to CIRP Journal of Manufacturing Science and Technology, (2014) 159-167.

[6] V.K. Astashev, V.I. Babitsky, Ultrasonic Processes and Machines, Springer, Berlin Heidelberg, (2007) 51-66. 